\title{
NEUROLOGICAL COMPLICATIONS OF LUMBAR SPINE PAIN SYNDROMES
}

\author{
Gustaw Wójcik ${ }^{1,2}$, Jolanta Piskorz ${ }^{3,4}$, Joanna Iłżecka ${ }^{4}$ \\ ${ }^{1}$ Diagnostic Imaging Department, The Zofia z Zamoyskich Tarnowska Regional Hospital in Tarnobrzeg. \\ ${ }^{2}$ Balneotherapy Department, Chair of Rehabilitation, \\ Physiotherapy and Balneotherapy at the Medical University in Lublin \\ ${ }^{3}$ Department of Anesthesiology and Intensive Care, \\ The Zofia z Zamoyskich Tarnowska Regional Hospital in Tarnobrzeg \\ ${ }^{4}$ Neurological Rehabilitation Laboratory, Chair of Rehabilitation, \\ Physiotherapy and Balneotherapy at the Medical University in Lublin
}

Wójcik G., Piskorz J., Iłżecka J. (2014) Neurological complications of lumbar spine pain syndromes. Health Problems of Civilization 2 (8), p. 10-14

\begin{abstract}
Summary: Back pain is a common health problem. Natural degenerative processes reduce its strength, causing symptoms of sciatica, numbness, paraesthesia with muscle weakness and sensory surface, leading to muscle atrophy, inclusive. Degeneration of the intervertebral disc and hernia formation in the first place causing damage to the spinal cord and nerves and spinal facet joints and ligaments become a source of pain spondylogenic. In the case of advanced degenerative changes of the axial movement of the camera conservative treatment is sometimes ineffective, and the chronic nature of the disease and its neurological complications diminish the quality of life and often lead to disability. In order to reduce the time the patient recovers minimally invasive procedures are used, the effectiveness of which can sometimes vary depending on the location of the source of the pain. PURPOSE OF THE WORK is to present the possible neurological complications resulting from chronic spinal pain syndromes. MATERIAL AND METHODS: Data were from the PubMed database of the last 20 years, presented in a descriptive analysis. CONCLUSIONS: lower back pain make a huge therapeutic difficulties.
\end{abstract}

$\underline{\text { Key words: }}$ back pain, spinal cord compression, radiculalgia, lumbar disc herniation

\section{Introduction}

Lower back pain is a very common health problem. Lack of physical activity, chronic stress, overweight and overloading the spine make back pain become one of the diseases of civilization. In addition to these environmental factors, another cause of back pain are natural degenerative processes within the intervertebral discs, which reduce the strength of the spine and reduce its load carrying capacity. The most common pain syndromes of backroot pain include lumbosacral pain (lumbalgia, sacralgia, radiculalgia).

The type of pain depends on the irritation of one of the two neural systems: core or nerve roots. Irritating these systems triggers the most common symptoms of sciatica, numbness or paresthesias in the extremities, muscle weakness and sensory superficial and leads to muscle atrophy.

Degeneration of the intervertebral disc is very common, and their incidence increases with age. This has important implications since degeneration of the intervertebral disc is very often a prelude to the herniated disc, which can cause damage to the spinal cord, spinal nerves, or both structures at the same time (Vangelder et al. 2013).

Most pathological changes concern the intervertebral disc and facet joints, but they may also be in the ligaments and muscles joints becoming a source of changes around spinal areas. Hypertrophy and fibrosis of the yellow ligament is one of the major causes of stenosis of the lumbar spine. Brown's scale identifies a number of reasons for lower back pain but because of the clinical symptoms of neurological complications spondylogenic and neurogenic causes are important. Spondylogenic ones are initiated by damage of the intervertebral disc due to osteo-articular changes. Neurogenic ones are the result of irritation of the nerve roots (Zhang et al. 2013).

\footnotetext{
Adress for correspondence: Gustaw Wójcik, Medical University of Lublin, Chodźki 6, 20-093 Lublin,

e-mail: gustaww@tlen.pl, phone: +48 (81) 7187503

Tables: 0, Figures: 0, References: 20, Full text PDF www.hpc.edu.pl Copyright (C) Pope John Paul II State School of Higher Education In Biała Podlaska, Sidorska 95/97, 21-500 Biała Podlaska Indexation: Index Copernicus, Polish Ministry of Science and Higher Education. This is an open-access article distributed under the terms of the Creative Commons Attribution Noncommercial License (http://creativecommons.org/licenses/by-nc/3.0), which permits use, distribution, and reproduction in any medium, provided the original work is properly cited, the use is non-commercial and is otherwise in compliance with the license.
} 
The course of the degenerative spine disease is associated with the development of a more or less persistent pain and complications such as damage to the nervous system in the immediate vicinity of the structural changes of the skeletal system. Osteoarthritis of the spine affects a large part of the population in its middle and old age. The chronic nature of the disease and its neurological complications diminish the quality of life and often lead to disability. Treatment and social care are associated with large financial output. To reduce costs and shorten the time of return of the patient to a full recovery, new techniques of minimally invasive treatments for the spine are developed. Their effectiveness largely depends on the correct qualifications for surgery, and on the correct choice of treatment for the patient. To meet the requirements of an effective procedure, the source of pain in the spine needs to be located, as well as the symptoms of dysfunction of the nerve roots need to be skillfully associated with the level at which there is compression of nerve tissue.

One of the most common sources of pain in osteoarthritis disease of the spine is intervertebral disc. The source of pain is mainly in damaged or irritated during loads and movements of the torso fibrous ring, innervated by the end of the sinus nerve cord, also known as reversible nerve ( $n$. sine-vertebralis vs $n$ reccurens). Pain can be provoked or worsened by changing body position or the burden on the spine, with the accompanying intensification of muscle tone, but without radicular syndrome. This kind of pain also increases whilest prolonged standing or sitting and is felt more in the lumbar region than in the lower limb.

Discogenic pain can be difficult to differentiate from pain coming from the facet joints. An important element of the differential nature is that the pain derived from intervertebral disc does not disappear after blocking the faced joints, while the introduction of physiological $\mathrm{NaCl}$ solution under pressure or a contrast medium to the intervertebral disc regenerates fibrosus pain.

Discography as a method in the field of diagnostic imaging allows for the presentation of the inner ring of fibrous joints resulting from cracking of the structure as a result of carrying excessive loads. Imaging methods such as CT or MRI allow for the morphological evaluation and determination of objective changes that can cause neurological deficits. Diagnostic imaging allows for making a decision as to the appropriateness of surgical intervention (Menon 2013).

As in the peripheral joints osteoarthritis disease, the disease process in the spinal area covers especially the facet joints despite the fact that the exit point of this condition is damage to the intervertebral disc. The result of these changes is the weakening of the supporting function of the spine and a destabilization of the intersegmentary links. Tolerance to mechanical stress is reduced, which sensitizes the formation of spine pain. An important role in the development of back pain is played by a richly innervated facet joint capsule traumatized during pathological vertebral sliding movements. Pain originating from the facet joints is dull, diffuse, located locally in the spine area and also may include a proximal portion of the limbs.

The pain is provoked by a change in body position, especially hyperextension, and is exacerbated as a result of mechanical burdens. Actions which are recommended in these cases, such as reducing the burden of the spine, orthotics, painkillers and non-steroidal anti-inflammatory drugs are not always effective, especially in the chronic pain associated with disorders of the spine stability. On the other hand surgical procedure - spinal fusion (fusion of rings) - is an invasive action changing the biomechanical functions of the spine: from the hypermobility of certain segments to completely excluding them from moving. This increases the load on adjacent segments, which take over the lost range of motion and are more exploited, and thus- are usually not free of the degenerative process. Hence the possibility of iatrogenic complications and recurrent pain.

Compression of the nerve tissue depending on the place of occurrence is associated with the presentation of different types of neurological disorders. One of them is caused by the assembly of the cone of the core terminal part of the pathology of spinal cord, called cone. The clinical picture of the syndrome consists of sensory disturbances in the area of skin around the perianal, perigenital areas and the inner surface of the thighs, dysfunctional voiding and sexual function and pain in the affected area. The anal reflex is absent (Campbell et al. 2005). So is the paresis. The etiology is most often traumatic, sometimes vascular.

\section{Purpose of the study}

The aim of the study is to present the possible neurological complications resulting from chronic pain syndromes of the lumbar spine. 


\section{Material and methods}

Method of descriptive analysis was applied. The study was conducted on the basis of an assessment of the PubMed literature from the years 1992 to 2013. The analysis covered the cases of patients with pain due to disc herniation of the lumbar spine as well as spinal stenosis and intervertebral holes causing compression of nerve tissue and an increased risk of neurological deficit. Cases which were analyzed concerned patients treated conservatively and patients undergoing surgical decompression of the nerve tissue.

\section{Discussion}

Damage to the intervertebral disc is the starting point for the formation of back pain syndromes. Lumbar spinal stenosis in addition to the neck part of the spine has the largest range of motion, therefore, it is particularly vulnerable to damage to the intervertebral discs. Compression of the intervertebral disc associated with its lowering affects the disorder of the entire mechanics of the motion segment. The resulting from this instability causes changes in load transfer from the upper part of the body by moving the axle load of a large area of the intervertebral discs to the relatively small outgrowths joints. Static disorders of the spine also bring about changes related to the morphology of the intervertebral holes causing stenosis. Reduction of light for the intervertebral holes conflicts with the tissue disrupting the function of the nerve roots and blood vessels. Such anatomical changes in motion segment induce adaptive changes in the adjacent segments of the movement affecting the working conditions of the nervous tissue as well as ligament and muscle (Wójcik et al. 2013)

Therapeutic treatments are designed to restore optimal anatomical components of bone , ligament and muscle conditioning the proper operation of the nervous tissue.

The lumbar segment of the spine is the source of more frequent pain, suffering and disability than any other part of the body. Pain in the lower back may be associated with the decompressing intervention procedures, such as, disk decompression, spinal fusion and vertebroplasty. The subdural steroid injections provide short-term and sometimes long-term pain relief. Percutaneous decompression of a disk is used to treat nerve root caused by intervertebral disc herniation. Spinal fusion is useful in the treatment of pain syndromes in the case of degenerative changes in the facet joints. Percutaneous vertebroplastics provides short-and long-term relief of pain in patients with diseases of the spine. However, careful selection of patients is essential to the success of any of these techniques (Gang et al. 1998).

Many researchers have tried to find a predictive method for assessing the effectiveness of treatment of disc herniation symptoms of sciatica. Rodet et al in their study used the preoperative electromyography (EMG) to assess the prognostic outcome of patients undergoing surgical treatment of intervertebral disc because of the oppression of the nerve roots at the lumbar level. Patients with normal EMG up to 73\% after surgery reported pain relief, while in patients with abnormal EMG paresthesias occurred twice as often and more often the relapses of irritation of the nerve roots occurred (Rodet et al. 1999).

Swartz et al reported a case of the patient with unilateral neurogenic calf hypertrophy caused by compression of the right S1 nerve root. Despite the development of neurogenic muscle hypertrophy patient's strength of the gastrocnemius muscle weakened. MRI test was conducted for the patient which confirmed right-sided damage to the intervertebral disc with compression of the S1 nerve root. Decompressing surgery was applied, after which the progressive nature of the symptoms was detained but muscular deficit remained. This confirms the hypothesis that the $\mathrm{S} 1$ nerve root dysfunction is a predisposing factor for the development of neurogenic muscle hypertrophy. It signifies that patients with symptoms of unilateral hypertrophy of the calf muscles require a thorough diagnostic evaluation focused on radiculopathy to determine the etiology of asymmetric neuromuscular conditions (Swartz et al. 2002).

Werle et al described also a hypertrophy of the gastrocnemius muscle in 52 - year-old man in the course of severe symptoms of sciatica. Researchers using EMG demonstrated that there are areas of damage, demyelinating innervated by the roots of S1 and S2. They confirmed the relationship between the occurrence of sciatica with hypertrophy of the muscles and at the same time their weakness (Werle et al. 1992).

Kim et al studied the differences in thickness of the transversus abdominis muscle between patients with chronic low back pain and the healthy ones. These studies were conducted on a group of muscles, the activity of which is associated with abdominal pressure yield, causing decrease in volume of the viscera and causing an increase in pressure in the abdominal cavity. This is very important in the mechanism supporting capacity of the lumbar spine, since this greatly reduces muscle tone back, and as a result reduces the size of the pressure level on the joints and intervertebral discs. In their conclusions, the researchers found that patients with chronic low back pain there often occurs transverse abdominal muscle atrophy and only the muscle reaction rate remains at a similar level as in healthy subjects. Disorders in the morphology of the abdominal muscles significantly affect the progression of the disease of the spine (Kim et al. 2013). 
Lenoir et al described a case of 42 - year old patient, who two years earlier had undergone spinal surgery due to stenosis of the spine and the emergence of the early symptoms of cauda equina. The operation was completed to the satisfaction from the perspective of disappearance of neurological symptoms. The patient however developed bilateral radiculopathy at L3 and L4 root level. To reduce the symptoms of pain the patient was given at a subdural level of intervertebral space L2/L3 steroids. Injection was madev without a damage to the dural sac and without any technical difficulties, however, soon after that the symptoms of paraplegia were observed in the patient. Emergency surgery was performed immediately to release the roots, however, surgical operation proved ineffective. The patient's neurological status remained unchanged. Four days later, the T2 MRI examination showed high signal intensity at the height of the cone of the core. Diagnosis was an ischemic cone. Probably as a result of injection of steroids parent artery supplying the area of the cone core has been damaged (Lenoir et al., 2008).

Bao et al described a case of 26 - year-old woman, who suffered a sharp pain around the thoraco-lumbar region accompanied by hypoesthesia of the right leg. MRI revealed spinal cone compression. The symptoms were unusual because radiculopathy instead of functional disorders of urination and defecation, typical of oppression of cone core presented an image of sciatica that can occur in the course of lumbar radiculopathy (Bao et al. , 2008).

Rougerie et al cite a case of 63 - year-old woman who was admitted to the neurosurgery department because of severe back pain radiating to the right lower limb. The pain appeared after exercise. Physical examination showed pain syndrome with hypoesthesia of the right spine of the anal area. The X-ray demonstrated widening of the intervertebral space L1/L2, which was interpreted as an expansive, intraspinal change. MRI found deformation and displacement of the cauda equina nerve roots by cyst. Due to adhesions of the cyst with a few roots surgical resection was incomplete resulting in persistent radicular pain returned and sphincter disorders remained. Excision of cysts is often incomplete and complicated by frequent risk of recurrence (Rougerie et al. 1999).

Pseudomeningocele usually occur after surgery of the cervical or lumbar spine segment. Although many of them remain asymptomatic, they can cause compression of the spinal cord and nerve roots. Macki et al described a case of 57 - year old woman who underwent laminectomy with discektomia, after which the patient experienced a significant weakness of the lower limbs and difficulty in walking. The conducted MRI revealed the presence of pseudomeningocele causing spinal cord compression. Positive neurological symptoms were caused by compression of the nerve tissue. Surgical treatment of anatomical changes in the spinal canal and epidural anesthesia may be a cause of iatrogenic pseudomeningocele causing myelopathic symptoms (Macki et al. 2013).

Rillardon et al conducted an analysis of long-term functional capacity after surgical treatment of lumbar spinal stenosis. Good results were obtained for the decompression of the nerve root, which was reflected in the cessation of symptoms of neurogenic claudication. Residual lumbalgia was a major problem at the last follow-up. The study found that the dominant factor influencing the functional outcome is the mental attitude of the patient. Other factors that affect the same population were continuing objective neurological disorders. For most patients, surgical treatment of spinal canal stenosis of the lumbar provides good long-term results and patient satisfaction, but the risk of another operation associated with persisting neurological disorders is $10 \%$ in the population of patients (Rillardon et al. 2003).

Also research was carried out based on the assessment of pain intensity before surgeries on stenosis of the lumbar spine using analogue scale VAS and disability scale Oswestry Disability Index (ODI).After decompression surgeries within 2 years after the procedure $31 \%$ of patients reported recurrence of back pain, and $54 \%$ of them reported further pain radiating into the lower extremities. The authors emphasize that the greater the intensity of back pain before surgery the weaker the effects of pain relief after decompression. (Sigmundsson 2013).

In some cases, when there are no anticipated effects of the treatment of chronic back pain and neck, stimulation of the spinal cord is applied. According to Podwal et al. neurostimulation procedure can not show a full effect, since about $40 \%$ of patients respond to it in a small degree(Podwal et al. 2013).

Also six cases were described of myelopathic oppression after lumbar surgery, in which patients have reported acute neurologic deterioration between 6 and 10 days after surgery. Patients reported progressive weakness of the lower limbs of a radiculopathy, or cauda equina injury (Fushimi et al. 2013).

Lykissas et al compared the efficacy of treatments using recombinant bone morphogenetic protein (BMP-2) to stabilize after the discektomia treatments using endogenous material. It was found, however, that treatments using BMP-2 are subject to an increased risk of neurological deficit (loss of sensory and motor function) due to the induction of bone formation at ectopic sites causing damage to nerve tissue (Lykissas et al. 2013).

Most neurological complications are an indication for the next operation, especially when associated with damage in and around nerve roots of the spinal cord. However, they may be irreversible, and sometimes dramatic. Common complications result from complications within the roots of the peripheral nerves and part of them concerns meningeal complications including paraplegia due to spinal complications. Currently, emphasis is placed on efforts to tackle the development of complications, which are particularly dangerous in terms of damage to the core. Their consequences are serious and their state depends on the severity of neurological syndrome. The use of less invasive techniques significantly reduces the incidence of these complications (Michel 1995). 


\section{Conclusions}

1. Pain in lower spine area causes enormous diagnostic and therapeutic difficulties.

2. Occurrence of pain not responding to conservative treatment should lead to considering the possibility of surgery, but also it does not give full guarantee of cure, as it increases the risk of complications.

3. The greater the severity of pain in the lumbar spine before surgery, the weaker the results of treatment and the more frequent complications.

\section{References:}

1. Bao XY., Ding XH., Lu YC. (2008), Sparganosis presenting as radiculalgia at the conus medullaris. Clin Neurol Neurosurg. 110(8): s. 843-846.

2. Fushimi K., Miyamoto K., Hioki A., Hosoe H., Takeuchi A., Shimizu K. (2013), Neurological deterioration due to missed thoracic spinal stenosis after decompressive lumbar surgery: A report of six cases of tandem thoracic and lumbar spinal stenosis. Bone Joint J. 95(10): s. 1388-1391.

3. Gangi A., Dietemann JL., Mortazavi R., Pfleger D., Kauff C., Roy C. (1998), CT-guided interventional procedures for pain management in the lumbosacral spine. Radiographics. 18(3): s. 621-633.

4. Kim KH., Cho SH., Goo BO., Baek IH. (2013), Differences in Transversus Abdominis Muscle Function between Chronic Low Back Pain Patients and Healthy Subjects at Maximum Expiration: Measurement with Real-time Ultrasonography. J Phys Ther Sci. 25(7): s. 861-863.

5. Lenoir T., Deloin X., Dauzac C., Rillardon L., Guigui P. (2008), Paraplegia after interlaminar epidural steroid injection: a case report. Rev Chir Orthop Reparatrice Appar Mot. 94(7): s. 697-701.

6. Lykissas MG., Aichmair A., Sama AA, Hughes AP., Lebl DR., Cammisa FP., Girardi FP. (2013), Nerve injury and recovery after lateral lumbar interbody fusion with and without bone morphogenetic protein-2 augmentation: $A$ cohort-controlled study. Spine J. s. 1654-1659.

7. Macki M., Lo SF., Bydon M., Kaloostian P., Bydon A. (2013), Post-surgical thoracic pseudomeningocele causing spinal cord compression. J Clin Neurosci. 5: s. 967-973.

8. Menon KV., Raniga SB., Al Busaidi AQ. (2013), MRI Morphology of surgically treated lumbar canal stenosis: $A$ retrospective study. J Spinal Disord Tech. 11(21) s. 276-281.

9. Michel CR. (1995), Neurologic complications of surgery for spinal deformities. Chirurgie. 120(11): s. 36-38.

10. Podwal J., Georgy MM., Georgy BA. (2013), Spinal cord stimulators in an outpatient interventional neuroradiology practice. J Neurointerv Surg. 10(22): s. 109-114.

11. Rillardon L., Guigui P., Veil-Picard A., Slulittel H., Deburge A. (2003), Long-term results of surgical treatment of lumbar spinal stenosis. Rev Chir Orthop Reparatrice Appar Mot. 89(7): s. 621-631.

12. Rodet D., Berthelot JM., Maugars Y., Prost A. (1999), Prognostic value of preoperative electromyography for outcome of lumbosacral radiculopathy of discal origin. Presse Med. 28(37): s.2031-2033.

13. Rougerie C., Chazerain P., Arthuis F., Chicheportiche V., Ziza JM. (1999) Spinal neurenteric cyst revealed by a cauda equina syndrome. Presse Med. 6;28(5): s. 229-230.

14. Sigmundsson FG., Jönsson B., Strömqvist B. (2013), Preoperative pain pattern predicts surgical outcome more than type of surgery in patients with central spinal stenosis without concomitant spondylolisthesis: A register study of 9051 patients. Spine (Phia Pa 1976), 10(29): s. 3451-3457.

15. Swartz KR., Fee DB., Trost GR., Waclawik AJ. (2002), Unilateral calf hypertrophy seen in lumbosacral stenosis: case report and review of the literature. Spine (Phila Pa 1976). 27(18): s.406-409.

16. Vangelder LH., Hoogenboom BJ., Vaughn DW. (2013), A phased rehabilitation protocol for athletes with lumbar intervertebral disc herniation. Int J Sports Phys Ther. 8(4): s. 482-516.

17. Werle C., Kuntz JL., Ruh D., Mohr M., Wiederkehr JP., Bloch JG., Asch L. (1992), Sciatic radiculalgia with muscular hypertrophy. Rev Rhum Mal Osteoartic. 59(9): s. 567-570.

18. Campbell WW., DeJong RN., Haerer AF. (2005), DeJong's the neurologic examination, Ed. 6. Lippincott Williams \& Wilkins, s. 485-486.

19. Wójcik G., Bulikowski W., Piskorz J., Stawińska T., Sokołowska B. (2013), Computed tomography and stretch tests in diagnosing sciatica as a method for ensuring patient well-being. Health and wellness. Sci. ed. Wiesław Kurlej, Lublin, NeuroCentrum. s. 223-235.

20. Zhang K., Sun W., Liu XY., Zhao CQ., Li H., Sun XJ, You-Zhuan X., Ding W., Zhao J. (2013), Hypertrophy and Fibrosis of the Ligamentum Flava in Lumbar Spinal Stenosis is Associated with increased Expression of LPA and LPAR1. J Spinal Disord Tech. 11(15) s. 511-519. 